\title{
A Bayesian risk assessment of the COVID-19 pandemic using FMEA and a modified SEIR epidemic model
}

\author{
Yacine Koucha* and QingPing Yang \\ Department of Mechanical and Aerospace Engineering, Brunel University London, Uxbridge, UK
}

Received: 2 December 2020 / Accepted: 16 May 2021

\begin{abstract}
The COVID-19 outbreak is of great concern due to the high rates of infection and the large number of deaths worldwide. In this paper, we considered a Bayesian inference and failure mode and effects analysis of the modified susceptible-exposed-infectious-removed model for the transmission dynamics of COVID-19 with an exponentially distributed infectious period. We estimated the effective reproduction number based on laboratory-confirmed cases and death data using Bayesian inference and analyse the impact of the community spread of COVID-19 across the United Kingdom. We used the failure mode and effects analysis tool to evaluate the effectiveness of the action measures taken to manage the COVID-19 pandemic. We focused on COVID-19 infections and therefore the failure mode is taken as positive cases. The model is applied to COVID-19 data showing the effectiveness of interventions adopted to control the epidemic by reducing the reproduction number of COVID-19. Results have shown that the combination of Bayesian inference, compartmental modelling and failure mode and effects analysis is effective in modelling and studying the risks of COVID-19 transmissions, leading to the quantitative evaluation of the action measures and the identification of the lessons learned from the governmental measures and actions taken in response to COVID-19 in the United Kingdom. Analytical and numerical methods are used to highlight the practical implications of our findings. The proposed methodology will find applications in current and future COVID-19 like pandemics and wide quality engineering.
\end{abstract}

Keywords: Coronavirus / COVID-19 / Bayesian inference / SEIR model / stochastic epidemic models / FMEA / failure mode and effect analysis

\section{Introduction}

The coronavirus disease 2019 (COVID-19) is an infectious disease and global pandemic caused by severe acute respiratory syndrome coronavirus 2 (SARS-CoV-2), and has been designated a Public Health Emergency of International Concern by the World Health Organization (WHO) [1]. The 1918-19 H1N1 influenza pandemic was the last time the world responded to an imminent global disease outbreak on the size of the current COVID-19 pandemic with no exposure to vaccines. The COVID-19 disease was first detected in Wuhan, China in 2019, and has since spread worldwide, leading to a coronavirus outbreak of 2019-20 [2]. The virus is suspected to have an animal origin by spillover infection and was first transmitted to humans in Wuhan, China, in November or December 2019 and became a major outbreak by early January 2020 . The United Kingdom's COVID-19 pandemic hit the nation at

\footnotetext{
* Corresponding author: yacinekoucha@live.com
}

the end of January 2020. There is often a delay between the onset of symptoms and correct diagnosis. The most critical things are the prompt diagnosis and identification of the infected, and the number of confirmed patients. Common symptoms include coughing, shortness of breath and fever, while less common symptoms may include muscle pain, sputum problems, and sore throat. Although most cases have mild symptoms, some cases have progressed to extreme pneumonia and multi-organ failure.

We seek to estimate the number of new infections estimated to stem from a single case around the world. A useful measure used to test viral transmissibility is the basic reproduction number, $\mathcal{R}_{0}$, which measures the number of secondary cases in a highly susceptible population due to each index case because there is no authorised vaccine available [3]. Fraser et al. [4] defines $\mathcal{R}_{0}$ as the condition of being contaminated or immunised (naturally or by vaccination) by no other individuals. This value is determined from the inherent characteristics of a disease, such as how quickly it is transmitted from one person to the next, along with human behavioural elements 
that influence how often sick and susceptible people come into contact. $\mathcal{R}_{0}$ is proportional to the contact rate and will vary according to the local situation. If $\mathcal{R}_{0}>1$, then epidemics take off, perhaps necessitating renewed lockdowns or other interventions, the pathogen is able to enter a susceptible population, and then, on average, each infected person produces more than one new infected individual. If $\mathcal{R}_{0}<1$, then the number of cases is decreasing and expected to die out, possibly allowing societies to open back up [5]. For example, if $\mathcal{R}_{0}$ is 3 , then it is expected that one person with the disease will, on average, infect three others. The value and scope of $\mathcal{R}_{0}$ during an outbreak can be calculated at different times. It can be estimated at the beginning of an outbreak (initial reproduction number) or at any time during the outbreak (time-dependent reproduction number).

In certain cases, however, not all contacts would be susceptible to infection and the average number of secondary cases per infectious case will be less than $\mathcal{R}_{0}$. Instead, this is measured by another quantity: the effective reproductive rate $\left(\mathcal{R}_{t}\right)$. The magnitude of $\mathcal{R}_{t}$ can be determined by the product of $\mathcal{R}_{0}$ and the portion of the exposed population $N$ that is susceptible $S$, so $\mathcal{R}_{t}=\mathcal{R}_{0} \times S / N$. Since $S / N \approx 1$, we can assume that $\mathcal{R}_{t} \approx \mathcal{R}_{0}$, thus simplifying the mathematics.

In this paper, we present a modified SEIR model for the transmission dynamics of COVID-19 in order to estimate $\mathcal{R}_{t}$ to the pandemic in the United Kingdom based on the data of confirmed cases identified by Bayesian inference. The objectives of this article are:

- To study the risks of COVID-19 infections and to evaluate the effectiveness of the action measures taken to manage the COVID-19 pandemic by combining Bayesian inference and failure mode and effects analysis (FMEA) together.

- To assess the impact of English government protective measures to COVID-19 by comparing the empirical and posterior statistics of $\mathcal{R}_{t}$ at different time periods with and without the measures being implemented.

- To apply these ideas to future pandemics and in wider quality engineering applications.

This study will show that, combined with FMEA and conditional analysis, Bayesian modelling will help to expand and provide responses to this outbreak.

\subsection{The epidemic theory}

Many articles have been published on the importance of the estimation of reproduction numbers in recognising the epidemic and possible threat of COVID-19 [6-8]. Various other approaches were used to assess the reproduction numbers to varying degrees of success, such as, the exponential growth (EG) method, maximum likelihood estimation (MLE) and the recursive Bayesian estimation (RBE) [9]. Reproduction number data helps us to evaluate which control measure is most effective in reducing the value of $\mathcal{R}_{t}$ to zero. For public health services, this will help provide valuable guidance on COVID-19. In one study provided by the Imperial College London COVID-19 Response Team [10], an $\mathcal{R}_{t}$ estimate of 2.4 was used to match the epidemic's early growth rate in Wuhan, but may vary between 2.0 and 2.6. In another study [11], scientists predicted that each infected person in the United Kingdom will, on average, infect about 2.5 other people in 5 days, then go on to infect 406 in total (after 35 days from exposure); but if there is a reduction in social contact (i.e. "social distance"), it is believed that the rate of infection can be reduced significantly to just 15 infections after 35 days. This was supported by the data and empirical evidence in the previous study [10]. Here, their estimates indicate that a combination of case isolation, social distancing of the entire population and either household quarantine or the closing of schools and universities are needed to reduce $\mathcal{R}_{t}$ below 1 . It was presumed that the rate an individual gets infected is treated as a gamma random variable with mean 1 and shape parameter equal to 0.25 . In another study published online in Emerging Infectious Diseases [12], the $\mathcal{R}_{t}$ had risen to a median of 5.7. That is about double an earlier $\mathcal{R}_{t}$ estimate of 2.0 to 2.6. An analysis of 12 modelling studies shows that the mean $\mathcal{R}_{t}$ for COVID-19 is approximately 3.28 , with a median of 2.79 (May 2020). This is in line with the estimates of $\mathcal{R}_{t}$ from Italy, which range from 2.76 to 3.25 . The introduction of mitigation and social distancing measures related to the COVID-19 pandemic has been reported to decrease $\mathcal{R}_{t}$ [13]. Between May and September 2020, the value of $\mathcal{R}_{t}$ across many European countries varied around 1 . As at 31 October 2020, the $\mathcal{R}_{t}$ value across the United Kingdom has stabilised between 0.9 and 1.2 .

\subsection{Data source and coverage}

Real-time data on the number of laboratory-confirmed cases, recoveries and deaths due to COVID-19 have been derived from a variety of authoritative sources, such as the Official Websites of Ministries of Health or other Government Agencies and the Social Media Pages of Government Authorities. Most of the data can also be accessed via Worldometer (https://www.worldometers.info/coronavi rus/\#countries), which is a reference website and has been cited in more than 10,000 published books and 6000 technical journal articles and has been named one of the best free reference websites of the American Library Association (ALA), the oldest and largest library organisation in the world. Data on hospitalisation for the average length of stay in hospitals in many countries around the world can be found in the OECD (https://data.oecd.org/ healthcare/length-of-hospital-stay.htm). Data on infections and testing, hospital resource use, mask use and social distancing (as well projections and forecasting) is provided by the Institute for Health Metrics and Evaluation (https://covid19.healthdata.org/). The data on these websites is collected and analysed regularly around the clock, 24 hours a day and 7 days a week.

We then follow the standard routine of dynamic modelling by focusing on the epidemic of COVID-19 in some of the most affected countries of Europe (i.e., United Kingdom, France, Spain, Netherlands, Germany and Portugal). We are interested in the daily and total number of lab-confirmed cases, recoveries and deaths. The latest cases data in the United Kingdom (as at 31 August 2020) is 


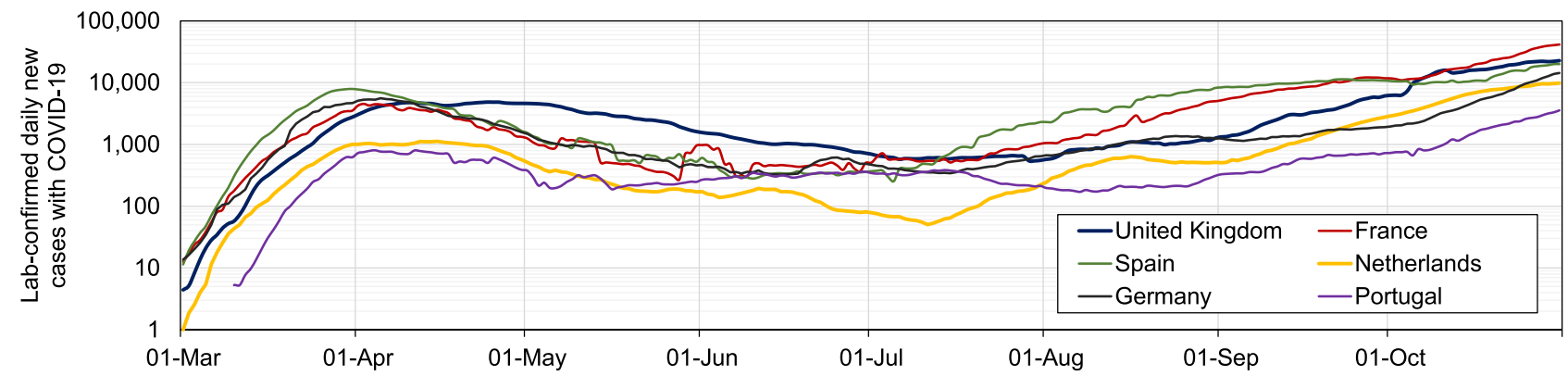

Fig. 1. A line graph for the number of lab-confirmed daily cases with COVID-19 for some of the infected countries in Europe. The number of cases are displayed using a logarithmic scale to base 10. Coverage: 1 March to 31 October. Data source: https:// ourworldindata.org/coronavirus/.

available for selected regions in the United Kingdom, whereas the latest death data is limited to the nations of the United Kingdom (i.e. England, Northern Ireland, Scotland and Wales). Figure 1 presents the number of lab-confirmed daily cases with COVID-19 for some of the infected countries in Europe, recorded between 1 March and 31 October, inclusive. The recovery data for Spain and the United Kingdom is unavailable to the public.

In view of the large volume of COVID-19 journal articles (more than 104,000 manuscripts identified via the Summon search at Brunel University as of 16 December 2020 for the key word 'COVID-19'), this analysis is focused on the community spread of COVID-19 in the United Kingdom between 1 March 2020 and 31 October 2020 inclusive, and on the impact of English government protective measures to COVID-19.

References, articles and websites for coronavirus data (including age, gender, incubation period, confirmed cases, deaths and recoveries) can be found in [14-17]. Additional articles published in IJMQE on the literature review and risk analysis of COVID-19 RT-PCR testing and at the same time, reliability analysis of diagnostic tests based on a combination of the methods of Fishbone Diagram and FMECA (Failure Mode, Effects and Criticality Analysis) can be found in $[18,19]$.

\section{Model and methods}

\subsection{A modified SEIR model adapted to COVID-19}

The classical SEIR (susceptible-exposed-infective-recovered) model assumes that births and deaths are not related to factors other than the disease itself, and is an extension to the classical SIR (susceptible-infective-recovered) model [20]. Each compartment in the SEIR model represents:

- $S$ : absolute number of susceptible individuals (those who may potentially contract the disease). Without further information, this group is represented by the whole population.

- E: absolute number of exposed individuals (those who have been infected but are not yet infectious).

- I: absolute number of infective individuals (those capable of transmitting the disease).

$-R$ : absolute number of recovered individuals (those who have become immune).
The SEIR epidemic model has been widely used in large populations to study the dynamics of infectious diseases when there is an incubation period during which individuals have been infected but are not yet infectious themselves. For this period, the individual is in the exposed state $E$. This may be called a latent phase, and may be infectious, partially infectious or not infectious yet. In the classical SEIR model, compartment $R$ also includes the number of people who died from the disease.

Several authors have developed different models of the classical SEIR taking into account the complexities of the disease in order to make the model as realistic as possible. To generalise the SEIR model to describe the COVID-19 pandemic in several countries, we adopt an additional compartment: $Q$ (quarantined), which adds a passage for the fraction of infectious individuals into the quarantined compartment. A characteristic feature of this model is that the total population in a given region $N$ is equal to the sum of all compartments:

$$
S(t)+E(t)+I(t)+Q(t)+R(t)=N .
$$

Equation (1) holds if the number of births $A$ are balanced with death rates $\mu$ during the time span of the disease (e.g. $A=\mu N)$. These quantities are expressed in units of births and deaths per 1000 individuals per year, respectively. All individuals in each compartment are subjected to a natural death. We define the reciprocal $\mu^{-1}$ as the average life expectancy of a country's population in a given year. This value applies to typical human deaths (e.g. due to natural death, usual influenza, fatalities, etc.) and is not linked to COVID-19.

Initially, we may assume that the population at time $t=0$ only includes susceptibles, so that, $S(0) \approx N, E(0) \geq 0$, $I(0) \geq 0, Q(0)=0$ and $R(0)=0$. There is a disease free equilibrium (DFE) with

$$
(S(0), E(0), I(0), Q(0), R(0))=(N, 0,0,0,0) .
$$

The number of individuals in $I$ tends to zero as $t \rightarrow \infty$, which ensures that $\lim _{t \rightarrow \infty} S(t)=: S_{\infty}>0$. Individuals in state $R$ have no further role to play in the epidemic, and we assume that infected individuals are mutually independent and shows an exponential growth, characteristic of any epidemic's initial stages [21]. 


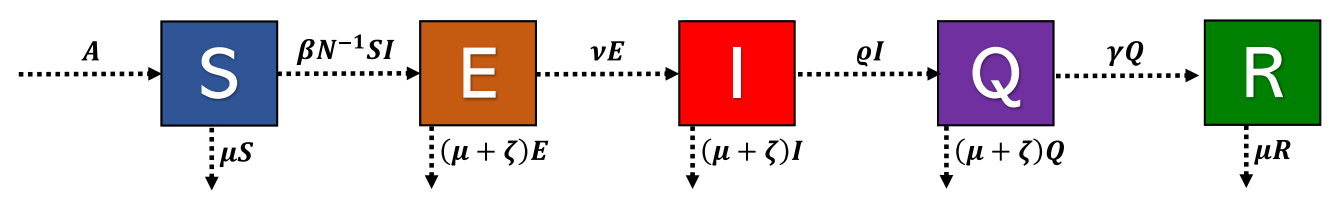

Fig. 2. A modified SEIR transmission model in which the boxes and the arrows represent the different compartments and transition rates used for COVID-19.

\subsection{Transition rates of vital dynamics}

The exponential distribution plays a significant role as the probability distribution that underlies the time spent in a compartment (or state), which is fundamental for Bayesian modelling (later sections will explain this). As such, these states can be seen as a flow diagram in which the boxes and the arrows represent the different compartments and transition rates of vital dynamics used for COVID-19 (a graphical illustration of the transmission model for COVID-19 can be seen in Fig. 2).

The modified SEIR model for COVID-19 described in Figure 2 can also be expressed by the following set of nonlinear ordinary differential equations (ODEs), which evolves according to:

$$
\begin{gathered}
S^{\prime}(t)=A-\left(\beta N^{-1} I(t)+\mu\right) S(t), \\
E^{\prime}(t)=\beta N^{-1} S(t) I(t)-(\nu+\zeta+\mu) E(t), \\
I^{\prime}(t)=v E(t)-(\varrho+\zeta+\mu) I(t), \\
Q^{\prime}(t)=\varrho I(t)-(\gamma+\zeta+\mu) Q(t), \\
R^{\prime}(t)=\gamma Q(t)-\mu R(t),
\end{gathered}
$$

where $\beta$ is the infection (or contact) rate, $v$ is the average incubation rate, $\varrho$ is the average hospitalisation rate, $\gamma$ is the recovery rate and $\zeta$ is death rate due to COVID-19.

The transition between each state can be explained as follows. Transmission of diseases occur through interactions between susceptible and exposed persons in the home, workplace, school, hospitals or at random in the neighbourhood, with the latter depending on the spatial distance between interactions. Any such contact results in the susceptible contracting the virus and thus becoming exposed and then infected, leading to transition $S \rightarrow E$ with probability $S(t) / N$ and population $\beta I(t)$ which exposes $\beta^{-1}$ new individuals per day. When exposed individuals transition from $E \rightarrow I$, the probability is 1 since all exposed individuals will become infected, the population is $E(t)$ and the latency rate is $v$ with a mean incubation period of $v^{-1}$.

The infected individuals eventually transition from $I \rightarrow Q$ which matches the "active confirmed cases" as documented in many official databases and reports. In fact, an infected person is not quarantined immediately because the authorities have often been unable to test enough people while keeping pace with the spread of the disease. The rate at which the number of infectious individuals move into the $Q$ compartment is given by $\varrho$, which is the inverse of the mean time needed to quarantine an infected patient. Recovered individuals do not return to compartment $S$ as long-term immunity is assumed, but it remains to be seen if patients recovered from COVID-19 can produce antibodies and gain long term immunity.

In most transitions, the duration is typically explained by an exponential distribution. The need to quarantine is driven by the number of confirmed cases and is necessary to significantly and effectively reduce the spread of infection. Individuals in compartments $E, I$ or $Q$ may die from the virus with rate of $\zeta$, respectively.

\subsection{Deriving $\mathcal{R}_{t}$ using the next generation method}

The next-generation matrix (NGM) approach was introduced in 1990 by Diekmann et al. [22] and is, in such situations, a general method of deriving the reproduction number $\mathcal{R}_{t}$, encompassing any scenario in which the population is divided into independent, disjoint variables. This technique assumes that the transmission probabilities between compartments are constant and that the distribution of the time of residence in each compartment is exponentially distributed. The NGM approach has been expanded on in numerous articles [23-25].

In this section, we outline the steps required to find the NGM operator in matrix notation [26], and then apply this approach to our modified SEIR model adapted to COVID-19.

Let $\mathbf{F}$ be the matrix of transmissions of new infections in the infectious compartments, and let $\mathbf{V}$ be the matrix of individuals entering and leaving the infectious classes (i.e. matrix transitions). Assuming that both matrices meet the conditions of $\mathcal{R}_{t}$ for compartmental models at the DFE from (2), with $S=N$, then we can form a NGM operator from the ODEs of the infectious classes, given by $\mathbf{F V}^{-1}$ where $\mathbf{V}^{-1}$ is the inverse $\mathbf{V}$. If we allow infected states to be denoted by indices $i$ and $j$, then entry $F_{i j}$ is the rate at which individuals in state $j$ give rise to individuals in state $i$. In other words, $F_{i j}$ is equal to zero if no new cases generated by a infectious individual in state $j$ can be in infected in state $i$ immediately after infection. The value of $\mathcal{R}_{t}$ is obtained from the spectral radius (dominant eigenvalue) of $\mathbf{F V}^{-1}$ [27].

Since we are concerned with the populations that transmit the virus, we may derive an expression for $\mathcal{R}_{t}$ from two cases: one with quarantine and one without it.

\subsubsection{Case I: No quarantine $(\varrho=0)$}

In this example, we only model the exposed $E$ and infected $I$ classes. Assuming that the DFE conditions are met at 
matrices $\mathbf{F}$ and $\mathbf{V}$, then the infectious class dynamics for $\mathcal{R}_{t}$ is given by

$$
\mathcal{R}_{t}=\frac{\beta v}{(\nu+\zeta+\mu)(\gamma+\zeta+\mu)}
$$

\subsubsection{Case II: Quarantine $(\varrho \neq 0)$}

For the second case, we take into account all three infectious compartments: $E, I$ and $Q$. Once again, assuming that the DFE conditions are met at matrices $\mathbf{F}$ and $\mathbf{V}$, then the infectious class dynamics for $\mathcal{R}_{t}$ may also be given by

$$
\mathcal{R}_{t}=\frac{\beta \nu \varrho}{(\nu+\zeta+\mu)(\varrho+\zeta+\mu)(\gamma+\zeta+\mu)} .
$$

Since classical statistics are empirical and see probability as something that has to do with a limiting frequency based on an observable proportion; it will then be more important to see how the subjective nature of the Bayesian method is implemented by using priori beliefs to describe a distribution of probabilities on the possible values of the unknown model parameters of the epidemic. Knowing the value of $\mathcal{R}_{t}$ makes this quantity very important for estimating the proportion of a population to be quarantined to avoid a further outbreak. In this case, our main interest will be to infer some of these unknown parameters, namely, $\beta, \gamma$ and $\zeta$, which will allow us to the predict size of the outbreak from $\mathcal{R}_{t}$ and apply control methods to reduce it. Other transition parameters (i.e. $v, \varrho$ and $\mu$ ) will be estimated empirically from known sources.

\subsection{Failure analysis}

The earliest form of risk analysis involved identifying all potential risks without taking into account the likelihood of them occurring. In the $1940 \mathrm{~s}$, structured risk analysis became popular as a risk assessment tool. Traditional risk analysis (TRA) approaches were plagued by poorly defined steps, high levels of uncertainty, and decision-making problems in the process. As a result, in the late 1940s, the US Armed Forces introduced failure mode effects analysis (FMEA) which is a significant improvement over TRA and acts as a proactive tool for identifying, evaluating, and preventing process failures. In the late 1950s, reliability engineers further improved this method to analyze issues that could arise from military device malfunctions. Today, there are a wide variety of risk analysis methods from which to choose: FMEA and its extension FMECA (Failure Mode, Effects, and Criticality Analysis), FTA (Fault Tree Analysis) and its extension ETA (Event Tree Analysis), DRBFM (Design Review by Failure Mode), HA (Hazard Analysis) and What-if/Checklist. The most widely used tools in the industry, however, are FMEA and FTA [28].

\subsubsection{Brief comparison of FMEA and FTA}

The FMEA is an inductive and bottom-up reasoning tool for failure analysis and is a core task in many areas of engineering (i.e. quality engineering including reliability and safety). FMEA necessitates a thorough review of each failure mode to understand how it could fail and the impact of such a failure on the process as a whole. By including a criticality analysis, the FMEA can be extended to an FMECA. The strictly qualitative FMEA can be made more quantitative in this way. As a quantitative analysis, a systematic approach to the use of FMEA/FMECA consists of identifying ways in which failure can occur ("failure mode"), then identifying the causes, effects of each failure mode, evaluating the corresponding risks, and then taking the necessary steps to minimise the risks and consequences. In the FMEA, the criticality of each failure mode is calculated using a risk priority number (RPN). The outcomes of an FMEA/FMECA are usually reported in a table (examples of tables and RPNs can be seen in greater detail in Sect. 4.4). There are standards available that provide guidance for conducting a proper FMEA/FMECA (e.g., [29]).

FTA is a top-down analysis that visually represents a failure path or failure chain and is based on the principle of Boolean logic, which allows for the construction of a set of True/False statements. The aim of FTA is to efficiently detect causes of failure and reduce risks before they happen. Both procedures involve a significant amount of time to fully implement. Moreover, a strictly qualitative FTA can be transformed into a quantitative FTA by incorporating quantitative component reliability data (e.g., failure rates) [30]. Using boolean algebra, such a quantitative FTA can be used to calculate the system's reliability. A comprehensive description of mostly quantitative FTAs is also given in [31]. As a result, potential failure modes can go undetected. To address these flaws, it is suggested to use: - FMEA as a complete analysis;

- FTA as a complete analysis;

- FMEA and FTA as a combined approached.

However, in the case of pandemics, we will show that the FMEA as a complete analysis can be used to assess the impacts of various potential failures in order to determine which prevention measures are most needed. Therefore in this paper, we extend these ideas to analyse the COVID-19 pandemic in the United Kingdom.

\subsubsection{The FMEA procedure}

The set up for our FMEA procedure is shown in Figure 3.

We define our process as the community spread of COVID-19 in the United Kingdom. Our failure modes consist of imported infected cases, local infected cases, infected items and isolated infected sites in different parts of the United Kingdom. Potential effects include higher mortality rates due to COVID-19, increased isolation effects on the well-being of individuals, higher reproduction numbers and a negative impact on the economy. Then we assess:

- How severe are the consequences of these failures? We denote these as the severity rating on a scale from 1 to 10 , where 1 is insignificant (or non-existent) and 10 is catastrophic.

- What is the likelihood of infected cases who tests positive? We denote this as the occurrence rating on a scale from 1 to 10 , where 1 is remote and 10 is inevitable 


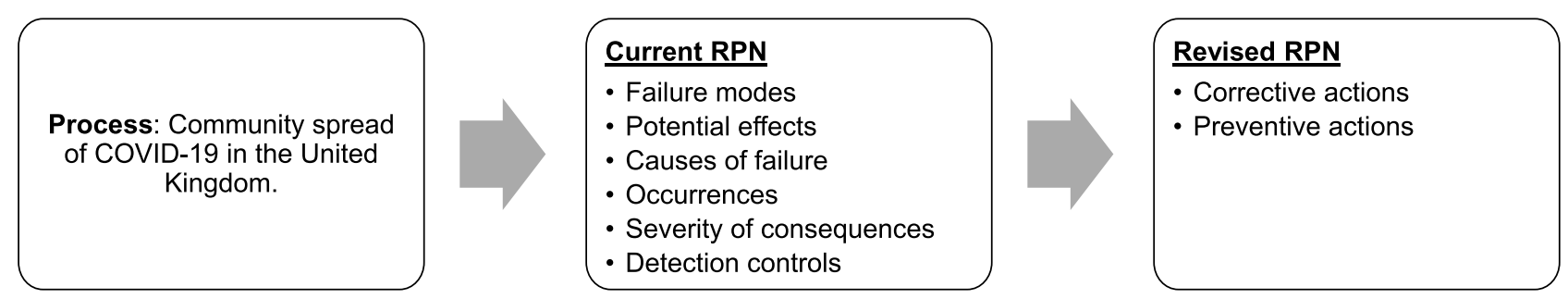

Fig. 3. FMEA mind map for the COVID-19 pandemic in the United Kingdom.

- What are the chances of detection of infected cases by the available control measures? We denote this as the detection rating on a scale from 1 to 10, where 1 means a control measure is highly certain to detect it and 10 means highly impossible (or no control exists).

We can then combine each of results above to obtain an overall rating for each failure mode. This value is called the Risk Priority Number (RPN) and is equal to

$$
\mathrm{RPN}=\text { Severity } \times \text { Occurrence } \times \text { Detection } .
$$

Another useful result is criticality which is found by multiplying severity by occurrence, i.e.

$$
\text { Criticality }=\text { Severity } \times \text { Occurrence. }
$$

Equations (10) and (11) gives relative priority to the failure modes in the order they should be addressed. A high RPN means that there are a lot of new cases and deaths and a corrective action may be needed for the occurrence, severity or detection of individual high ratings. This corrective action should be extended to containment and prevention methods such as country-wide lockdown, asymptomatic testing, sanitization of cities and towns, self-isolation and "stay at home" practices, social distancing, hand washing at regular intervals (provided by the NHS or other local health centres in accordance with government requirements), etc. Applying these methods should significantly reduce the previous RPN value to a smaller value closer to 1 . If the RPN reaches 1 , then the pandemic has likely ended.

\section{Bayesian method on infectious periods}

In this section, we consider how the Bayesian approach is applied to our modified SEIR model for infectious periods. Bayesian inference begins from the posterior probability as a result of two indicators: the "prior probability" and the "likelihood function" derived from the observed data. The prior probability is a central aspect of Bayesian inference and reflects knowledge on unknown parameters, which is combined with the likelihood function of new data to yield the posterior distribution. This is then used to make potential inferences and decisions involving each unknown parameter. Within such a model each parameter is believed to be independent of each other. The posterior distribution forms the heart of Bayesian inference.
Naturally, all Bayesian problems begin with Bayes' theorem:

$$
\pi_{*}\left(f\left(\mathcal{R}_{t}\right) \mid \mathcal{D}\right)=\frac{\mathcal{L}\left(\mathcal{D} \mid f\left(\mathcal{R}_{t}\right)\right) \pi_{0}\left(f\left(\mathcal{R}_{t}\right)\right)}{\mathbb{P}(\mathcal{D})},
$$

where $f\left(\mathcal{R}_{t}\right)$ is a function of the effective reproduction number $\mathcal{R}_{t}, \mathcal{D}$ is the data observed, $\pi_{0}\left(f\left(\mathcal{R}_{t}\right)\right)$ is the prior probability distribution for $f\left(\mathcal{R}_{t}\right)$ which represents our opinion and state of knowledge before observing the current data, $\mathcal{L}\left(D \mid f\left(\mathcal{R}_{t}\right)\right)$ is the likelihood distribution of $f\left(\mathcal{R}_{t}\right)$ associated with the model for the data as collected, and $\pi *\left(f\left(\mathcal{R}_{t}\right) \mid \mathcal{D}\right)$ is the posterior distribution for $f\left(\mathcal{R}_{t}\right)$ after we have computed everything on the right, taking into account the data we have observed. $\mathbb{P}(\mathcal{D})$ should be the probability of generating the data, but it is difficult to calculate on its own, so the alternative way of describing this relationship is to be one of proportionality:

$$
\pi_{*}\left(f\left(\mathcal{R}_{t}\right) \mid \mathcal{D}\right) \propto \mathcal{L}\left(\mathcal{D} \mid f\left(\mathcal{R}_{t}\right)\right) \pi_{0}\left(f\left(\mathcal{R}_{t}\right)\right) .
$$

Since the posterior function is a probability, the sum or integral over all possible $f\left(\mathcal{R}_{t}\right)$ should be 1 . In this case, the value of $\mathbb{P}(\mathcal{D})$ is the normalising constant which ensures that the posterior PDF integrates to unity and is given by:

$$
\mathbb{P}(\mathcal{D})=\int_{-\infty}^{+\infty} \mathcal{L}\left(\mathcal{D} \mid f\left(\mathcal{R}_{t}\right)\right) \pi_{0}\left(f\left(\mathcal{R}_{t}\right)\right) d f\left(\mathcal{R}_{t}\right):=C_{t} .
$$

In many epidemic cases, a distribution from the exponential family, namely the Poisson and Gamma distributions, fits the shape of the pandemic well. Our model consists of two layers: the likelihood of the data and the inference from prior to posterior on $\mathcal{R}_{t}$. The Bayesian method is therefore engaged in transforming time series of case numbers into probability distributions.

\subsection{Dynamic poisson cases}

Suppose we may estimate the infectious period to be exponentially distributed with mean $\gamma^{-1}$ and assume it to be constant throughout the duration of the COVID-19 pandemic. We may be interested in calculating $\mathcal{R}_{t}$, which provides a value on each day in the interval $t \in[1, T]$. We want to see today's posterior to be updated from yesterday's prior. In order to use the Bayes theorem, we must select a prior distribution. The flat or uniform prior is a very common choice for a prior distribution. A flat prior assumes that all probabilities on $f\left(\mathcal{R}_{t}\right)$ are equally likely. 
Since $\mathcal{R}_{t}$ could take any value from 0 to infinity, most of our beliefs about $f\left(\mathcal{R}_{t}\right)$ will come through data observation, i.e. the likelihood $\mathcal{L}$. In this case, the density function is uniformly distributed and therefore the prior function is proportional to 1 .

Suppose now that the rate of $j$ new cases per day can be described by a time-dependent Poisson distribution with parameter $\theta_{t} \equiv f\left(\mathcal{R}_{t}\right)$. By using Bayes' theorem from (12) and assuming a flat prior, the posterior probability of $\theta_{T}$ given seeing $j$ new cases at time $T$ is updated to

$$
\pi_{*}\left(\theta_{T} \mid j_{T}, \mathbf{j}, \theta\right) \propto \mathcal{L}\left(j_{T}, \mathbf{j}, \theta \mid \theta_{T}\right)=\prod_{t=1}^{T} \frac{\theta_{t}^{j_{t}} e^{-\theta_{t}}}{j_{t} !},
$$

where $\mathbf{j}=\left\{j_{1}, j_{2}, \ldots, j_{T-1}\right\}$ and $\boldsymbol{\theta}=\left\{\theta_{1}, \ldots, \theta_{T-1}\right\}$. For the sake of brevity, we define $\mathcal{D}:=\left\{j_{T}, \mathbf{j}, \boldsymbol{\theta}\right\}$. If $\theta_{t} \equiv \theta$, then the posterior $\mathrm{PDF}$ is $C_{T} \theta^{\sum_{t}} j_{t} e^{-T \theta}$ and $\theta_{T} \mid \mathcal{D} \sim$ Gamma $\left(\sum{ }_{t} j_{t}-1, T\right)$ distributed. Another useful representation of the above can be made by taking the natural log on both sides of the equation:

$$
\ln \pi_{*}\left(\theta_{T} \mid \mathcal{D}\right) \propto \sum_{t=1}^{T}\left\{j_{t} \ln \left(\theta_{t}\right)-\theta_{t}\right\} .
$$

Equation (13) is used in the subsequent sections.

\subsection{Bayesian inference on Bettencourt \& Ribeiro's $\theta$}

Bettencourt and Ribeiro [28] derived a relationship linking $\theta_{t}, \gamma, j_{t-1}$ and $\mathcal{R}_{t}$ together by:

$$
\theta_{t}=j_{t-1} \exp \left(\gamma\left(\mathcal{R}_{t}-1\right)\right)=: j_{t-1} \Psi_{1}\left(\mathcal{R}_{t}\right)
$$

Since the $\mathcal{R}_{t}$ value is dynamic and is more closely linked to recent values than older ones, we can follow Systrom's method [29] by considering the last $(w+1)$ days, that is $t \in[T-w, T]$. From the above, we can derive a posterior distribution for $\mathcal{R}_{T}$. By taking the last $(w+1)$ points in (14) and adapting Bettencourt \& Ribeiro's $\theta$ to that equation, the posterior probability is therefore

$$
\text { See equation (16) below. }
$$

From (16), we find that $\exp \left\{\sum_{t} j_{t} \ln \left(j_{t-1}\right)\right\}$ and $\exp \left\{\sum_{t} j_{t} \gamma\right\}$ are independent from $\mathcal{R}_{T}$. Since we are interested in $\mathcal{R}_{T}$, these terms may be taken out of the equation to become one with proportionality, i.e.

$$
\text { See equation (17) below. }
$$

Now, if we expand the summations in (17), we find that each $\mathcal{R}_{t}$ term for $t=T-w, \ldots, T-1$ is independent from $\mathcal{R}_{T}$. Therefore these excess terms can also be removed from the equation to become one with proportionality, thus reducing the posterior PDF to a more compact form:

$$
\pi_{*}\left(\mathcal{R}_{T} \mid \gamma, j_{T-1}, j_{T}\right)=C_{T}^{-1} \exp \left\{j_{T} \gamma \mathcal{R}_{T}-j_{T-1} e^{\gamma\left(\mathcal{R}_{T}-1\right)}\right\},
$$

where $C_{T}$ is the constant of integration after integrating the exponential function with respect to $\mathcal{R}_{T}$. Hence, $C_{T}$ is equal to

$$
\begin{aligned}
C_{T} & =\int_{0}^{\infty} \exp \left\{\gamma j_{T} \mathcal{R}_{T}-j_{T-1} \Psi_{1}\left(\mathcal{R}_{T}\right)\right\} d \mathcal{R}_{T} \\
& =\frac{\Gamma\left(j_{T}, j_{T-1} e^{-\gamma}\right)}{\gamma\left(j_{T-1} e^{-\gamma}\right)^{j_{T}}},
\end{aligned}
$$

where $\Gamma(a, b)$ is the upper incomplete gamma function with $\Gamma(a, b) \rightarrow 0$ if $b \rightarrow \infty$.

\subsection{Updated $\theta$ for the COVID-19 pandemic}

To find an expression that accounts for the evolution of new infections after each day in terms of epidemiological observables, we discretize the infectious ODE equations in (4), (5) and (6), for the change in daily number of cases between $t-1$ and $t$. Note that the cumulative number of exposures, cases and actively confirmed cases up to time $t$, $C E(t), C I(t)$ and $C Q(t)$, respectively. Epidemic reports most commonly give the frequency of cumulative infected cases, which are reported daily and given by $C I(t)-C I$ $(t-1)=\Delta C I(t) \approx C I^{\prime}(t)$. The change in daily cumulative

$$
\begin{aligned}
\pi_{*}\left(\mathcal{R}_{T} \mid \mathcal{D}\right) \propto \exp \left\{\sum_{t}\left(j_{t}\left(\ln \left(j_{t-1}\right)+\gamma\left(\mathcal{R}_{t}-1\right)\right)-j_{t-1} \exp \left(\gamma\left(\mathcal{R}_{t}-1\right)\right)\right)\right\} \\
\propto \exp \left\{\sum_{t} j_{t} \ln \left(j_{t-1}\right)+\sum_{t} j_{t} \gamma \mathcal{R}_{t}-\sum_{t} j_{t} \gamma-\sum_{t} j_{t-1} \exp \left(\gamma\left(\mathcal{R}_{t}-1\right)\right)\right\} . \\
\pi_{*}\left(\mathcal{R}_{T} \mid \mathcal{D}\right) \propto \exp \left\{\sum_{t} j_{t} \ln \left(j_{t-1}\right)\right\} \exp \left\{\sum_{t} j_{t} \gamma\right\} \exp \left\{\gamma \sum_{t} j_{t} \mathcal{R}_{t}-\sum_{t} j_{t-1} \exp \left(\gamma\left(\mathcal{R}_{t}-1\right)\right)\right\}, \\
\propto \exp \left\{\gamma \sum_{t} j_{t} \mathcal{R}_{t}-\sum_{t} j_{t-1} \exp \left(\gamma\left(\mathcal{R}_{t}-1\right)\right)\right\},
\end{aligned}
$$


exposures and quarantines are also given by $\Delta C E(t) \approx$ $C E^{\prime}(t)$ and $\Delta C Q(t) \approx C Q^{\prime}(t)$. We find that $C E(t), C I(t)$ and $C Q(t)$, between $t-1$ and $t$, obeys the following equations:

$$
\begin{gathered}
C E^{\prime}(t)=E(t) \approx \beta S(t) I(t) / N, \\
C I^{\prime}(t)=I(t) \approx \nu E(t), \\
C Q^{\prime}(t)=Q(t) \approx \varrho I(t) .
\end{gathered}
$$

Therefore, by substituting (19) into (20), the change in the cumulative number of daily cases updates to:

$$
C I^{\prime}(t) \approx v E(t) \approx \beta \nu S(t) I(t) / N .
$$

The RHS of equation (22) is exact if $S(t) / N$ is constant in the period $[t-1, t]$. Since $S(t) / N \approx 1$ and $\mathcal{R}_{0} \approx \mathcal{R}_{t}$, the ODE in (5) which models the change in $I(t)$ updates to:

$$
\begin{gathered}
I^{\prime}(t) \approx\{\beta v-(\varrho+\zeta+\mu)\} I(t), \\
=\left\{(\varrho+\zeta+\mu)\left((v+\zeta+\mu)(\gamma+\zeta+\mu) \mathcal{R}_{t} / \varrho-1\right)\right\} I(t),
\end{gathered}
$$

$$
=\left\{\phi_{1} \mathcal{R}_{t}-\phi_{2}\right\} I(t),
$$

where $\phi_{1}=\phi_{2}(\nu+\zeta+\mu)(\gamma+\zeta+\mu) / \varrho$ and $\phi_{2}=\varrho+\zeta+\mu$. The ODE in (24) was obtained by solving for $\beta$ in (9) and substituting it into (23). To obtain an equation which accounts for the change in daily new infections, we must integrate (25) between $t-1$ and $t$, which gives:

$$
I(t)=I(t-1) \exp \left\{\phi_{1} \mathcal{R}_{t}-\phi_{2}\right\}=: I(t-1) \Psi_{2}\left(\mathcal{R}_{t}\right) .
$$

Now, by rewriting the equation in terms of $\theta_{t}$ and $j_{t-1}$, we obtain $\theta_{t}=j_{t-1} \Psi_{2}\left(\mathcal{R}_{t}\right)$. Once again, by taking the last $(w+1)$ points in (14) and adapting the $\theta$ expression into our $\theta_{t}$ equation, the posterior probability is therefore:

$$
\pi_{*}\left(\mathcal{R}_{T} \mid \mathcal{D}\right)=C_{T}^{-1} \exp \left\{\phi_{1} j_{T} \mathcal{R}_{T}-j_{T-1} \Psi_{2}\left(\mathcal{R}_{T}\right)\right\},
$$

where

$$
C_{T}=\frac{\Gamma\left(j_{T}, j_{T-1} e^{-\phi_{2}}\right)}{\phi_{1} \exp \left\{-\phi_{2} j_{T}+j_{T} \ln \left(j_{T-1}\right)\right\}}
$$

\subsection{Prediction}

It may also be useful to have a better representation of the uncertainty in $\mathcal{R}_{t}$ via our posterior. Suppose we are interested in the next case $j_{T+1}$. We can use the posterior predictive distribution $\pi *\left(j_{T+1} \mid \mathcal{D}\right)$ which is the distribution of a new case $j_{T+1}$, marginalized over the posterior, i.e.

See equation (28) below. where $\Delta^{+} j_{T}=j_{T}+j_{T-1}$.

The data $\mathcal{D}$ is represented by the history of $\left\{j_{T}, j_{T-1}\right.$, $\left.\phi_{1}, \phi_{2}\right\}$. The posterior predictive distribution has the same mean as the posterior distribution, but a greater variance which takes into account the additional "sampling uncertainty" since we are drawing new data points. Often the $\pi *\left(j_{T+1} \mid \mathcal{D}\right)$ form can be obtained directly, but it is always simpler to analyze $\pi *\left(j_{T+1} \mid \mathcal{D}\right)$ using MCMC methods.

\section{Results}

In this section, we will test our Bayesian methods above applied to the coronavirus outbreak in Europe, namely, a model validation with selected countries (France, Germany, Italy and Portugal), and a Bayesian analysis according to the pandemic situation in the United Kingdom, to see what sort of outcomes we may observe. We recall that $\beta$ is the probability of transmitting the disease multiplied by the number of contacts per unit time. Essentially, the reduction of $\beta$ (and therefore $\mathcal{R}_{t}$ ) means that the peak decreases in intensity but shifts to later periods for $\mathcal{R}_{t}$ greater than 1 .

Data from the UK COVID Symptom Study indicates that while most individuals recover from COVID-19 within two weeks, one in 10 individuals may still have symptoms after three weeks and some may suffer for months. The current official estimated range for the recovery period is within 14 days $(1 / 14 \leq \gamma \leq 1)$, whereas the mean period of incubation observed appears to be $5-6$ days $(1 / 6 \leq v \leq 1 / 5)[30,31]$. In this case, $\gamma$ and $v$ could vary significantly among patients. For mathematical simplicity, we set the recovery and incubation rate to be the same and equal to $1 / 5$ (unless stated otherwise).

The results include parameter estimation, model validation, fatality analysis, the impact of governmental protective measures and outbreak forecasting. Integrals and other computations were computed in Mathematica and MATLAB, and converted to graphs and tables in Microsoft Excel.

\subsection{Parameter analysis and model validation for the COVID-19 pandemic for Western European countries}

Here, by comparing the Bayesian posterior averages with the actual data, we aim to justify the modified SEIR model using the posterior functions in (18) and (27) for forecasting the COVID-19 outbreak across selected countries in Western Europe. Example calculations for model (18) are given. We extract real-time data for the number of (smoothed) new cases from Ritchie et al. [32]. Here, we set $\gamma=1 / 5$ and $T=1, \ldots, 4$ to represent the period of $1-4$ March 2020. For simplicity, if we let $T=2$, then our posterior PDF in (18) reduces to $\pi_{*}\left(\mathcal{R}_{2} \mid \gamma, \mathbf{j}\right)=$ $C_{2}^{-1} \exp \left\{j_{2} \gamma\left(\mathcal{R}_{2}-1\right)-j_{1} e^{\gamma\left(\mathcal{R}_{2}-1\right)}\right\}$, where $C_{2}$ is a constant. Here, the value of $j_{1}$ and $j_{2}$ represents the number of

$$
\pi_{*}\left(j_{T+1} \mid \mathcal{D}\right)=\frac{\exp \left\{\phi_{2} j_{T+1}+j_{T} \ln \left(j_{T-1}\right)-\Delta^{+} j_{T+1} \ln \left(\Delta^{+} j_{T}\right)-\ln \left(j_{T+1} !\right)\right\}}{\Gamma\left(j_{T}, j_{T-1} \exp \left\{-\phi_{2}\right\}\right) / \Gamma\left(\Delta^{+} j_{T+1}, \Delta^{+} j_{T} \exp \left\{-\phi_{2}\right\}\right)}
$$


cases (after data smoothing) in times 1 and 2, respectively (i.e. the first two days of March). In this example, the focus is on the COVID-19 pandemic in two countries: Germany and Italy. For the case of Italy, we observed that $j_{1}=149.86$ and $j_{2}=222.43$, and so $C_{2}=2.97131 \times 10^{-59}$. The posterior mean, median, mode and variance are therefore found to be $\mathbb{E}\left[\mathcal{R}_{2} \mid \mathcal{D}\right]=2.963, \quad \mathbb{Q}_{0.5}\left[\mathcal{R}_{2} \mid \mathcal{D}\right]=2.975, \quad \mathbb{M}\left[\mathcal{R}_{2} \mid \mathcal{D}\right]=2.967$ and $\mathbb{V}\left[\mathcal{R}_{2} \mid \mathcal{D}\right]=0.113$, respectively, and the posterior probability that the epidemic will drop below 1 , given the last two days, is $1.86401 \times 10^{-8}$. The empirical $\mathcal{R}_{t}$ is the result of (15) when $\theta_{t}=j_{t}$ and $\mathcal{R}_{t}=\gamma^{-1} \ln \left(j_{t} / j_{t-1}\right)+1$. In this case, the empirical result of $\mathcal{R}_{2}$ is equal to 2.975 , which is strongly consistent with the posterior averages.

Table 1 presents the number of new cases in Germany and Italy over four days with the respective posterior means, medians, modes and other vital statistics. The posterior averages are in agreement with the empirical result.

The posterior PDF of this distribution, as well as three follow up cases in Italy, $j_{3}=258.14, j_{4}=311.43$, and $j_{5}=384.14$ with respective $\mathcal{R}_{T}$ for $T=3,4$ and 5 , have been illustrated in Figure 4.

The equation derived in (26) is an expression accounting for the evolution of new infections. Example calculations can be seen in Figure 5 for the variation of $\Psi_{1}\left(\mathcal{R}_{t}\right)$ and $\Psi_{2}\left(\mathcal{R}_{t}\right)$ for $\mathcal{R}_{t}$ with empirically estimated parameters describing COVID-19 outbreak in the United Kingdom. We find that $\Psi_{2}\left(\mathcal{R}_{t}\right)$ is in strong agreement with $\Psi_{1}\left(\mathcal{R}_{t}\right)$. These parameter values are related to different methods and should only be seen as a qualitative benchmark.

Example calculations on (27) are given in Table 2, which presents the number of new cases in four countries over three different periods. The respective posterior means, medians, modes, variances and $90 \%$ credibility intervals are given. For each period, we set $v=1 / 5.2$, $\varrho=1 / 7, \quad \gamma=1 / 5$ and $\zeta=0.15$. We then matched the empirical mortality rate to each country (e.g. $\mu=9.4 / 1000$ in the United Kingdom) so that $\phi_{1} \approx 7(0.293+\mu)(0.342+\mu)$ $(0.350+\mu)$. The posterior averages are strongly in line with the empirical outcome. The empirical result of $\mathcal{R}_{t}$ is the solution of (26) and is approximately equal to the posterior mode.

\subsection{Assessing the impact of English government protective measures to COVID-19}

The main objective of government protection policies and lockdowns is to reduce the rates of infection so as to prevent the health care system from being overloaded by too many serious cases at the same time. In order to research the impact of the protection measures adopted by the United Kingdom government, we compare the confirmed (relative) infection rate, the cumulative infections in a population $C I(t)$, the empirical reproduction number $\mathcal{R}_{t}$, and the posterior averages of $\mathcal{R}_{t}$, at different time periods with and without the measures being implemented. The National Health Service (NHS) is a publicly funded health care system in the United Kingdom. The primary responsibility for public health and healthcare in the United Kingdom rests with the Department of Health and Social Care

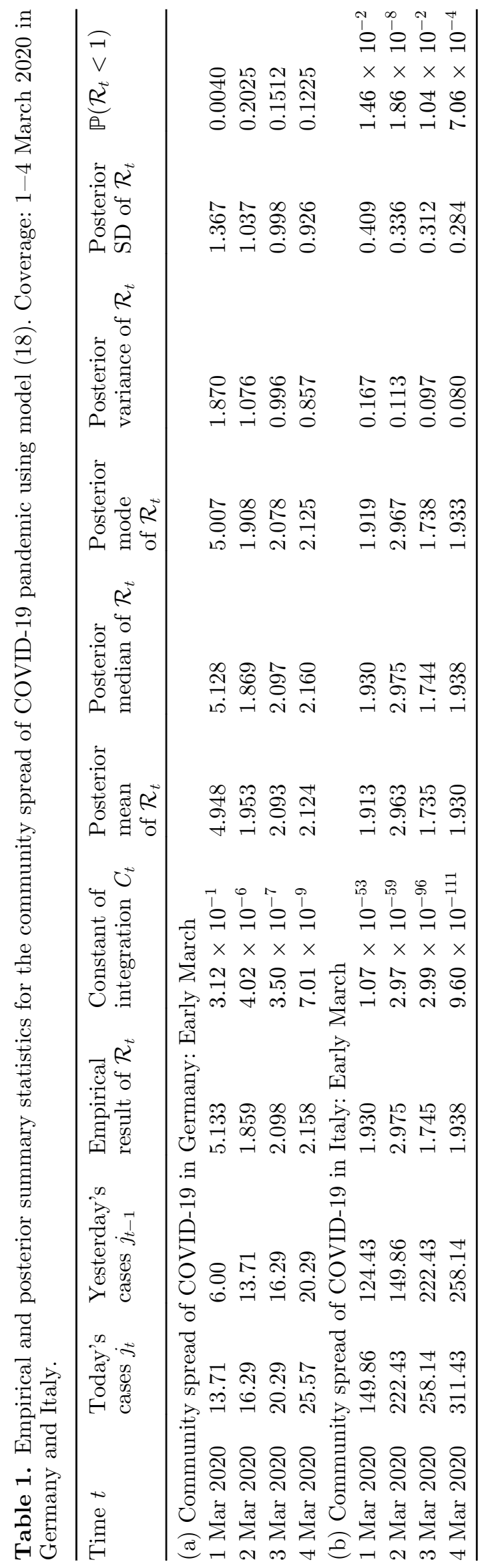




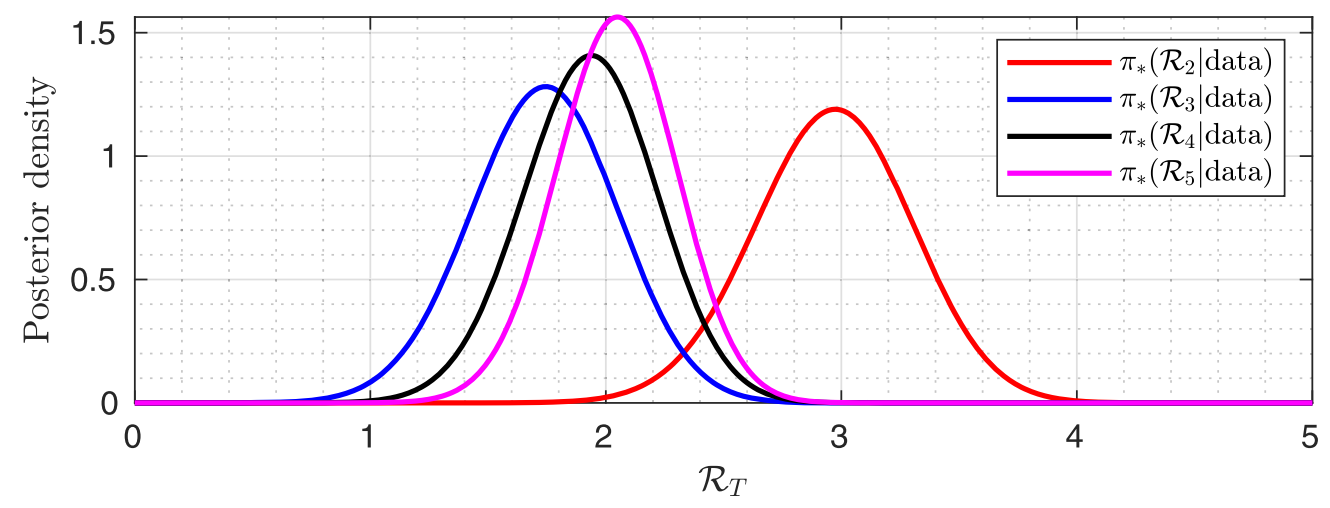

Fig. 4. Real-time data for the variation of $\pi *\left(\mathcal{R}_{T} \mid \mathcal{D}\right)$ for $\mathcal{R}_{T}$ over 5 days with $\gamma=1 / 5$. Coverage: Italy from $1-5$ March 2020 .
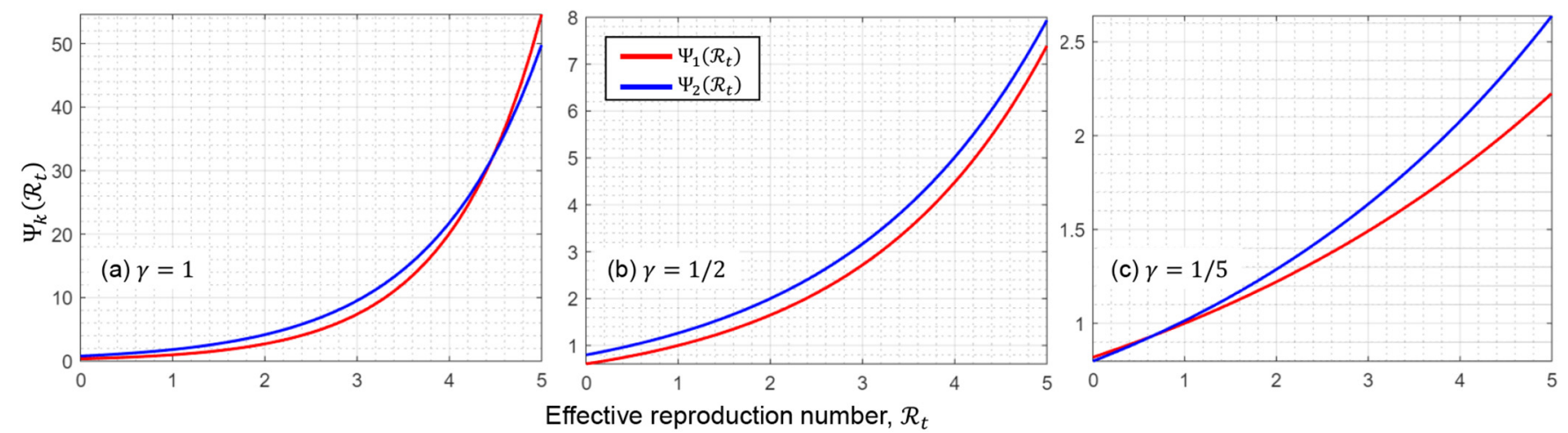

Fig. 5. The variation of $\Psi_{1}\left(\mathcal{R}_{t}\right)$ and $\Psi_{2}\left(\mathcal{R}_{t}\right)$ for $\mathcal{R}_{t}$ with empirically estimated parameters describing COVID-19 outbreak: $\gamma=\{1,1 / 2$, $1 / 5$ \} (both models), $v=1 / 5, \varrho=1 / 10, \zeta=0.116$ and $\mu=9.4 / 1000$. Coverage: $1-11$ September 2020 in the United Kingdom.

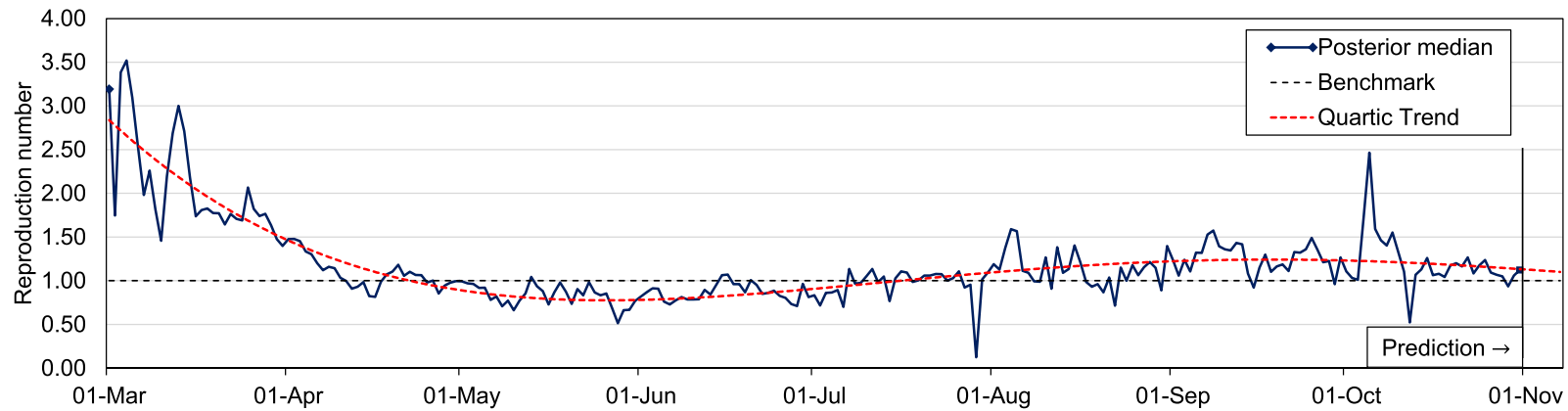

Fig. 6. The continuous red dotted lines refer to the predicted evolution in 7 days according to the modified SEIR model. Coverage: United Kingdom region, updated on 31 October 2020.

(DHSC). The periodic infection rate in the United Kingdom and how it varies over the course of time between early March and late October are shown in Table 3. The (relative) infection rate is expressed as the daily change in infections as a percentage of the value of the indicator in the earlier periods, i.e.

$$
\text { Relative infection rate }=\frac{\Delta I(t)}{I(t-1)}
$$

where $\Delta I(t)=I(t)-I(t-1)$. The comparison of the estimated rates of infection in the corresponding periods before and after the introduction of preventive measures highlights the significance and reliability of measures such as social distancing and lockdowns in managing and slowing down the spread of COVID-19. As of 31 October 2020 , there has been 989,745 and 42,592 confirmed cases of infection and death, respectively.

The forecast of the situation in the United Kingdom, based on the Bayesian method (Fig. 6), appears reasonably positive, as the (posterior median) $\mathcal{R}_{t}$ values follows a quartic $^{1}$ trend. Table 3 and Figure 6 are in strong

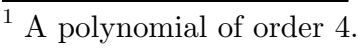


agreement with each other and represents the most probable scenario. According to a statistical study conducted in September 2020 [33], the number of positive cases doubles every seven to eight days, with a total of 3539 new coronavirus cases were recorded in the United Kingdom on 10 September. Scientists have estimated the empirical $\mathcal{R}_{t}$ value to be at 1.7 which appears to agree with our posterior estimates.

In countries where there is no public health intervention, such as Sweden, the demand for an intensive care unit (ICU), is estimated to be much higher than the intensive care capacity in other western countries, and as a result, there ends up being a much larger $\mathcal{R}_{t}$ number of deaths being predicted [34]. In the United Kingdom, the NHS is reported to have used half as many ICU beds as France, Belgium and other badly-hit European nations during height of pandemic in May 2020. This is equivalent to 50 infected patients attached to the ventilators for every million people in mid-April. In Belgium, where there were deaths at a similar rate to the United Kingdom at the time, the figure was approximately 111 per million people. During the same week, France treated 104 people per million in intensive care [35]. With 2.7 hospital beds per 1000 citizens, the United Kingdom has fewer hospital beds than other European nations, compared with an EU average of 5.2 [36].

\subsection{Fatality analysis}

In order to slow down the outbreak and therefore prevent an increase in deaths, social distances and other preventive measures were implemented in the United Kingdom around 23 March 2020. The daily deaths in the United Kingdom as well as the relative shift in deaths are shown in Figure 7 . The relative change in deaths mirrors equation (29) and is given by $\Delta D(t) / D(t-1)$ where $\Delta D(t)=D$ $(t)-D(t-1)$.

Another formula of interest in epidemiology is the naïve case fatality rate (CFR), which is the proportion of COVID-19 cumulative deaths over the total number of confirmed cases over a specified time period. This formula provides an overview of the severity of the disease and is given as:

$$
\mathrm{CFR}=D(t) / I(t) .
$$

For this study, we apply the above formula in (30) and present a fatality analysis that is important for government protection decision-making. Simple calculations using data recorded in the United Kingdom indicate that on 16 April, the CFR initially increased sharply and peaked at an alltime high of $21.5 \%$, and has since decreased with varying degrees of drops (with a few significant increases around June and July). The CFR rate fell below the "safety net" ( $2 \%$ level) for the first time on 4 August and has since remained below that level. The daily CFR rate in the United Kingdom is 0.3 percentage points lower than the global average as of 31 October 2020, at $1.0 \%$ and $1.3 \%$, respectively.

Infection, reproduction and fatality rates (see Tab. 3 and Fig. 7) depend on the preventive measures in place, the 


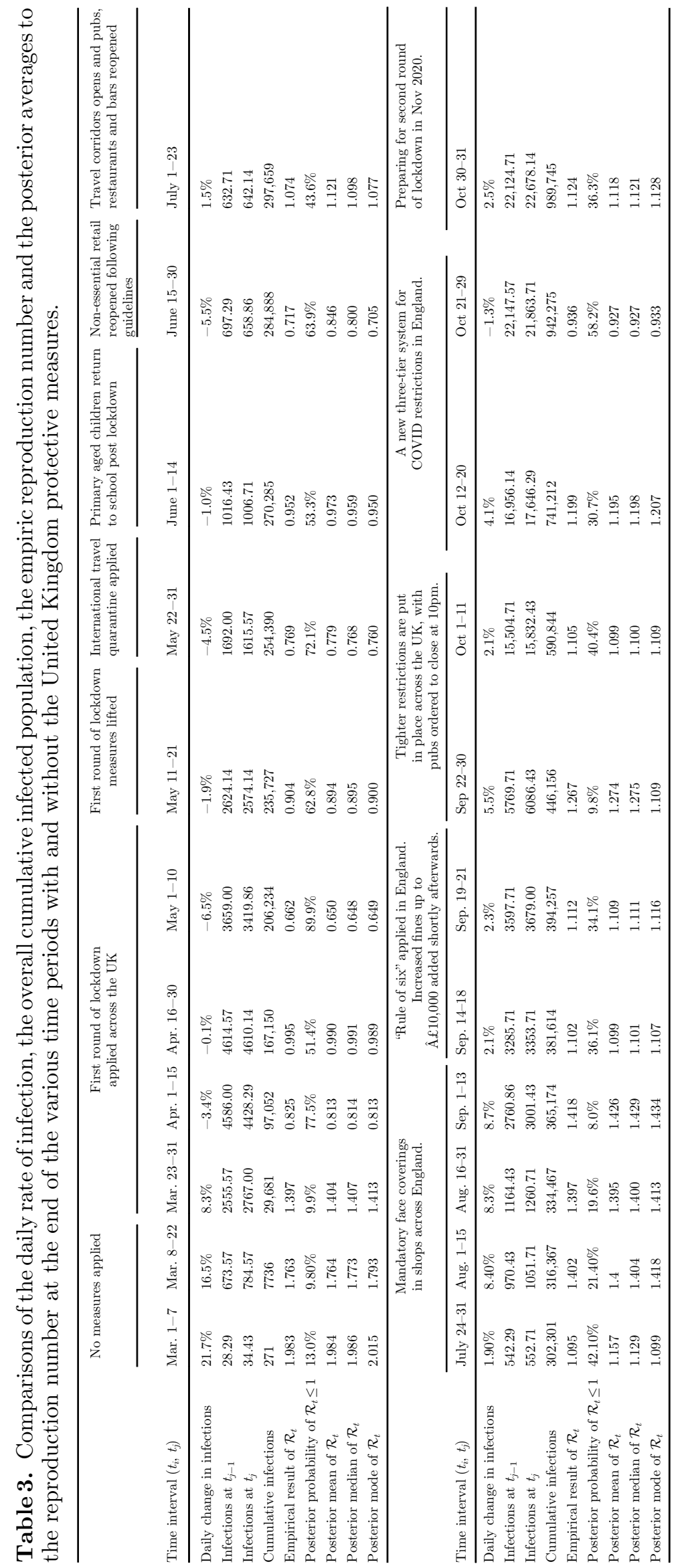



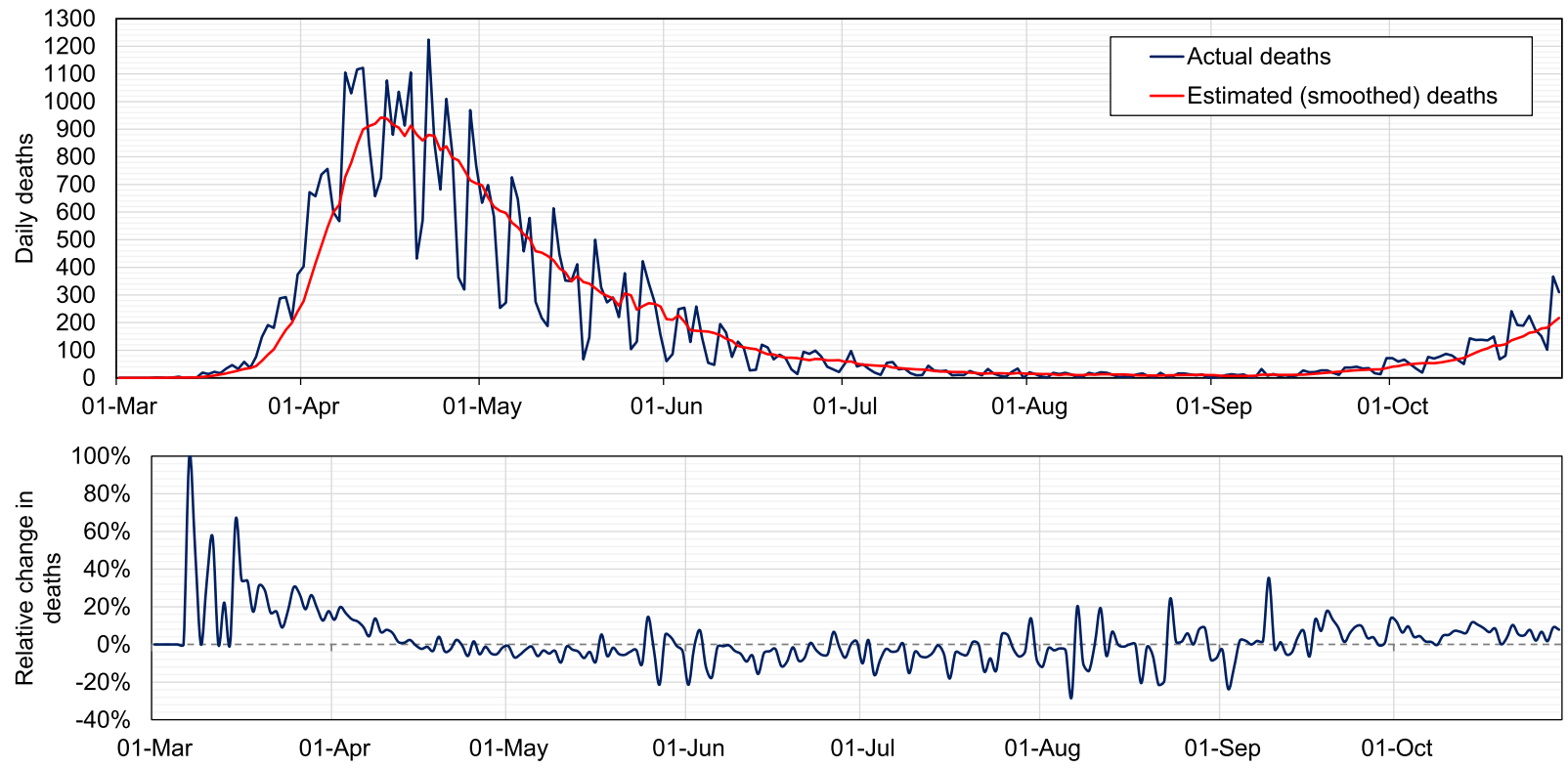

Fig. 7. The daily deaths (top) and the relative shift in deaths (bottom) in the United Kingdom.

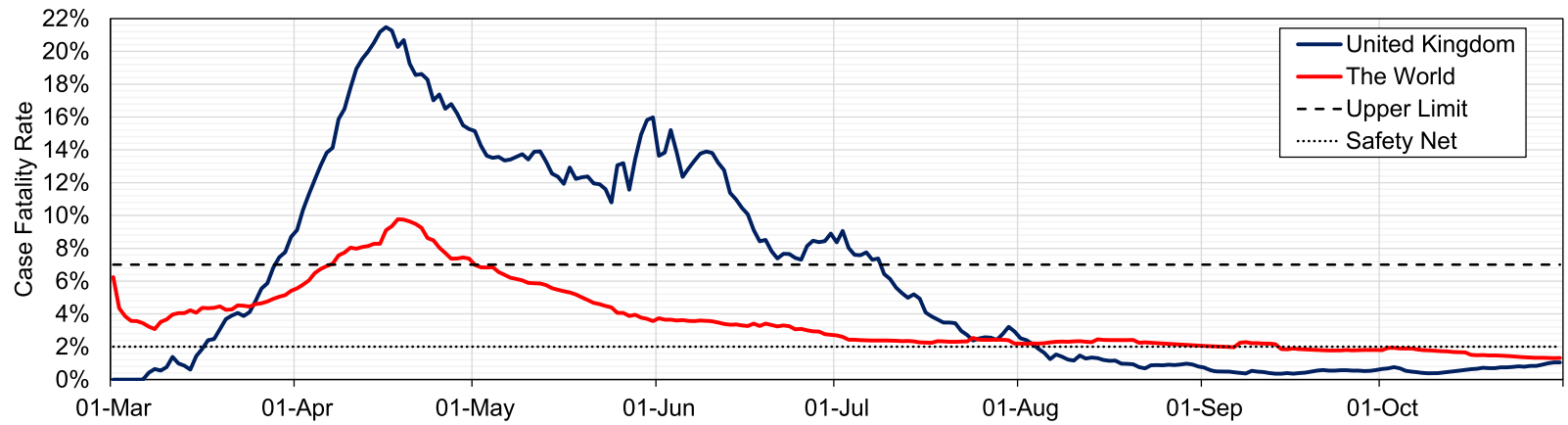

Fig. 8. Case fatality rate (CFR) in the United Kingdom and the world calculated using equation (28).

number of fatalities may rise due to a large number of infected individuals if the government safety measures are ignored. The rate of infection has been higher in care homes than in the community and there has been significant regional variation in the severity of the outbreak. This, in fact, reduces the number of ICU beds available as the number of intensive cases rises drastically.

\subsection{FMEA of the community spread of COVID-19 in the United Kingdom}

The number of COVID-19 cases, fatalities and reproduction numbers have an impact on the population's wellbeing and on the economy as a whole. An FMEA could be used to shed some light on the direction the government has taken and inform the future decision-making. Control measures such as national lockdown or selfisolation are effective in reducing the Detection rating (i.e. the probability for the failure to reach the individual) and thus reducing the severity of case fatalities and decreasing the Rate of Occurrence of positive infections in order to prevent failures. To demonstrate the use of an FMEA, a 10-point severity, occurrence and detection scale to represent the community spread of COVID-19 in the United Kingdom is presented in Table 4.

Each criterion is ranked from 1 (insignificant/remote/ failure prevented) to 10 (critical/extremely high/no detection opportunity). For the analysis of the community spread of COVID-19 in the United Kingdom (from March to October 2020), we matched Criteria S (CFR rate) to Figure 8, Criteria O (posterior median of $\mathcal{R}_{t}$ ) to Figure 6, and Criteria D (control methods) to the government policies defined in Table 3 for the given time intervals. The RPN and criticality values are calculated using equations (10) and (11), respectively. For the sake of brevity, we scaled criticality by a factor of 10 to match the RPN value. A graphical summary of our findings on the severity, occurrence and detection of COVID-19 throughout the United Kingdom (from 1 March to 31 October) is provided in Figure 9.

In our FMEA, we focused on the COVID-19 infections and thus the failure mode is taken as positive cases. In addition, the process phase, cause of failures, current 


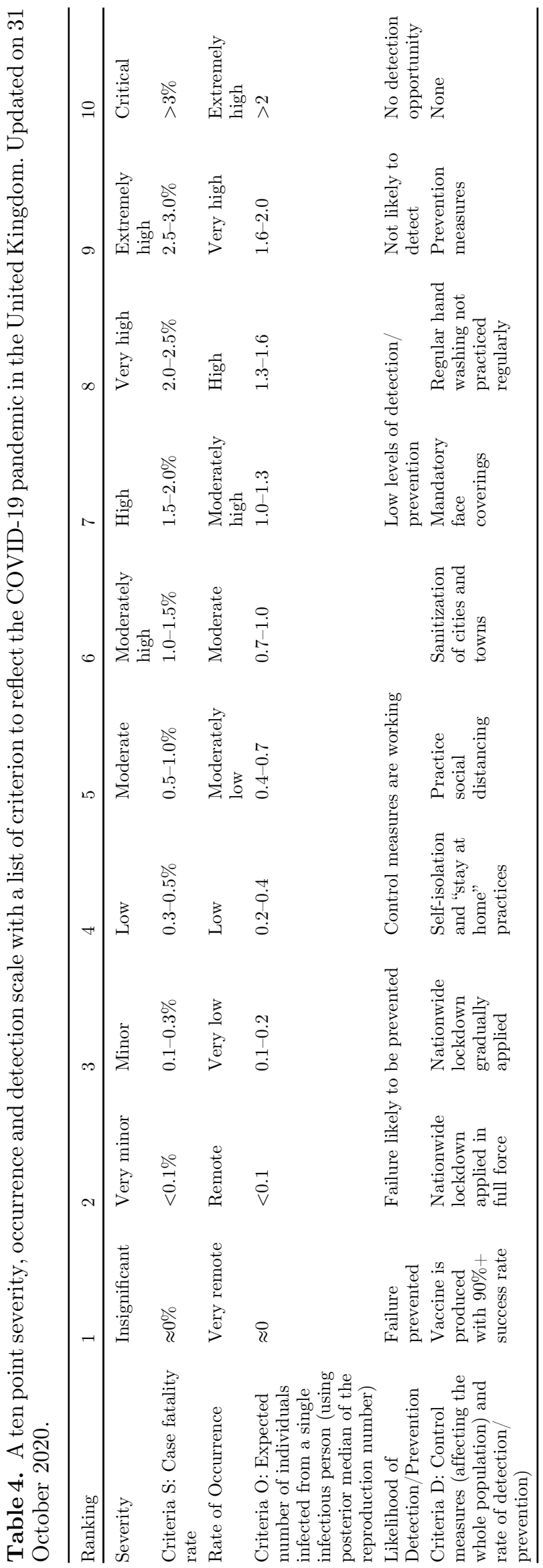

control measures and risk measures are summarised in Table 5, with the subsequent government responses and reevaluated risk measures are presented in Table 6 .

It is estimated that some of the main causes of the failure were government responses, namely, failure to shut down international airlines due to imported infections from outside the UK (22 Jan-27 Feb), no social distancing for more than 15 minutes at distances of less than 2 metres (28 Feb-23 Mar), delayed lockdown against the rising pandemic (23 Mar-4 May), failure to practice safety measures once travel corridors opens and pubs, shops, restaurants and bars had reopened (5 May-31 May), failure to follow policies and protocols set by the government (1 June-5 Sep) and overcrowded restaurants during the month-long 'Eat Out to Help Out' scheme and the re-opening of schools (6 Sep-31 Oct). The effect of failure for each process phase is discussed in the following paragraphs.

The failure to shut down international airlines affected the United Kingdom critically in the first process phase, as the national risk level for COVID-19 was raised from "very low" to "low" by Public Health England (PHE) on 22 January. As a result of this failure, British nationals had to move from Wuhan to quarantine at Arrowe Park Hospital. All airports in the United Kingdom were required to provide written guidance to unwell travellers. With no current measures were in place, the first two cases of COVID-19 were confirmed in the United Kingdom on 31 January.

In the second process phase, failure modes were the first deaths and early spread of the disease across the country. The effect of these failures has led to the slow spread of COVID-19 across the country. The number of confirmed cases rose to 23 on 29 February, after 10,483 people had been tested and two of the most recent cases had recently returned from Italy, while the third had returned from Asia. Subsequently, the United Kingdom's death toll was 55 , with the number of cases of COVID-19 passing 1500 by 16 March. The increased severity was due to the very high death rates that occurred.

In the third phase, the national lockdown came to full effect across the country and all non-essential shops, libraries, places of worship, playgrounds and outdoor gyms were closed by 23 March. The government's failure to respond strongly to the rising pandemic had raised the risk level from "moderate" to "high". Too much attention was also given to hospital deaths, not including those in care homes or the home of a person. The effect of these previous failures has led to a large increase in deaths, bringing the total number of deaths to 1019 by 28 March; 17,089 individuals tested positive. Following the lockdown, there was a significant increase in anxiety and depression among the UK population and the number of people infected in the hospital exceeded 10,000 by 31 March. In addition, less than a week later, the death toll was more than 5000 and the total number of reported cases was almost 52,000 on 6 April. An additional 823 deaths were reported on 21 April, which amounted to a total of 17,337 , a sharp increase in previous days, but many of them were related to deaths that occurred in previous days and weeks, some of which date back to March. 

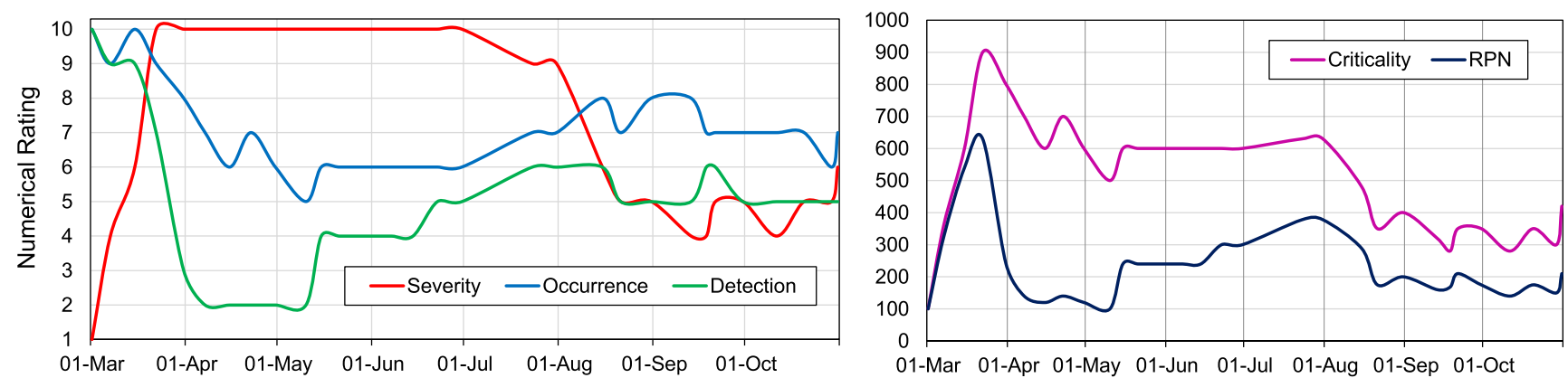

Fig. 9. A visual summary of a 10-point FMEA rating (left) and the criticality/RPN (right). Results reflect the United Kingdom COVID-19 pandemic by following the timeline on Table 3 and the evaluation criteria on Table 4 .

Following the easing of lockdown measures and continued restrictions in the fourth phase, the government's scientific guidance suggested that the $\mathcal{R}_{t}$ number had risen marginally from 0.5 to 0.9 at the end of the lockdown to $0.7-1.0$ on 15 May, closer to the point at which infections would begin to escalate exponentially. Fittingly, these values are in line with our posterior median estimates of $0.66-0.96$ and $0.88-0.94$, respectively. Restrictions continued and local lockdowns were introduced in the fifth phase as infection and death rates continued to rise. Wearing face masks were made compulsory for public transport (15 June), in shops and supermarkets in Scotland (10 July) and England (24 July) as well as indoor settings, such as cinemas and places of worship (31 July). These regulations may have significantly contributed to the reduction of the failure associated with the practise of safety measures once travel corridors and non-essential venues were reopened. However, the daily number of new cases exceeded 1000 on 9 August for the first time since June, rising from 1062 to 310,825 , but it is not apparent whether the increase is due to higher infection rates or higher test volumes.

In the last phase, many of the main causes of the failure were government responses from the fifth phase, namely overcrowded restaurants during the month-long 'Eat Out to Help Out' scheme in August and the re-opening of schools in England, Wales and Northern Ireland for the autumn term. The effect of these failures is likely to have led to a significant increase in cases since September. We find that an additional 2988 cases were reported on 6 September, the highest number since 22 May, with an estimated a posterior median of $\mathcal{R}_{t}$ of 1.32 . As government researchers warn that the virus is widespread across the nation, the $\mathcal{R}_{t}$ number floated between $1.1-1.4$ on 18 September, which is consistent with our posterior median estimate of $1.10-1.30$. There were 6178 new cases on 23 September, the highest daily reported since 1 May, and the posterior median of $\mathcal{R}_{t}$ was 1.32. Cases continue to rise and, three weeks later, on 16 October, there were 27,900 new cases in England, a $60 \%$ increase over the previous week, while the reported $\mathcal{R}_{t}$ was between $1.3-1.5$ and our posterior median estimate of $\mathcal{R}_{t}$ was between $1.26-1.55$. Finally, the United Kingdom crossed a million cases of COVID-19 on 31 October, taking 21,915 cases to 1,011,6600.

\section{Conclusion and discussion}

In this paper, we have considered Bayesian inference for the modified SEIR model with an additional state (selfquarantined $Q$ ), which characterizes the pandemic of COVID-19, focusing in particular on $\mathcal{R}_{t}$ in cases where the infectious period is exponentially distributed and where the posterior densities are extended from a Poisson-gamma mixture. The choice of exponential infectious period is used because it provides a natural analogue to the deterministic differential equation models, in which the constant removal rate corresponds to the exponentially distributed infectious period.

We have combined Bayesian inference and FMEA together to study the risks of COVID-19 infections and to evaluate the effectiveness of the action measures taken to manage the COVID-19 pandemic. The Bayesian model and FMEA are applied to the COVID-19 data showing the effectiveness of the interventions adopted to control the pandemic by reducing the $\mathcal{R}_{t}$ of COVID-19. In our FMEA, we focused on COVID-19 infections and therefore the failure mode is taken as positive cases. The process phases, cause of failures, current measures and current risk ratings are discussed, and subsequent government action measures are presented with re-assessed risk ratings.

The results have shown that the combination of Bayesian inference, compartmental modelling and FMEA are effective to model and study the risks of the COVID-19 transmissions, able to evaluate quantitively the action measures and identify the lessons learned from the impacts of governmental measures and actions carried out in response to the community spread of COVID-19 in the United Kingdom. Here, we have justified the use of the SEIQR model over the SEIR by comparing the previous posterior model in (18) with the improved model in (27) for forecasting the COVID-19 outbreak across selected countries in Western Europe, by comparing the Bayesian posterior averages with the actual data. The equations, $\Psi_{1}\left(\mathcal{R}_{t}\right)$ and $\Psi_{2}\left(\mathcal{R}_{t}\right)$, are functions of the posterior models in (18) and (27), respectively. We found that $\Psi_{2}\left(\mathcal{R}_{t}\right)$ is in strong agreement with $\Psi_{1}\left(\mathcal{R}_{t}\right)$, which is effectively seen as a qualitative benchmark for describing COVID-19 outbreak in the United Kingdom (see Fig. 5). We have then shown 


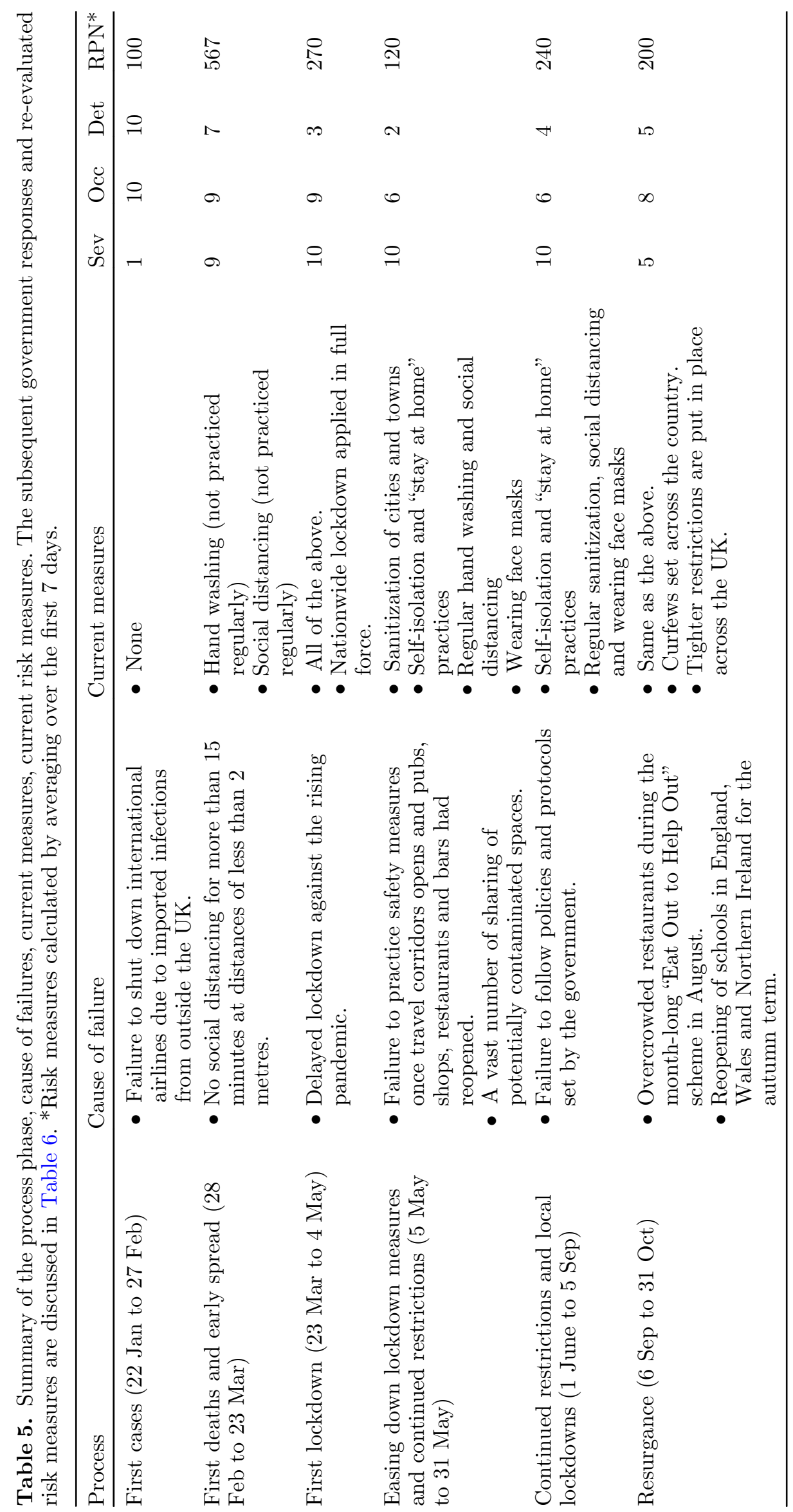




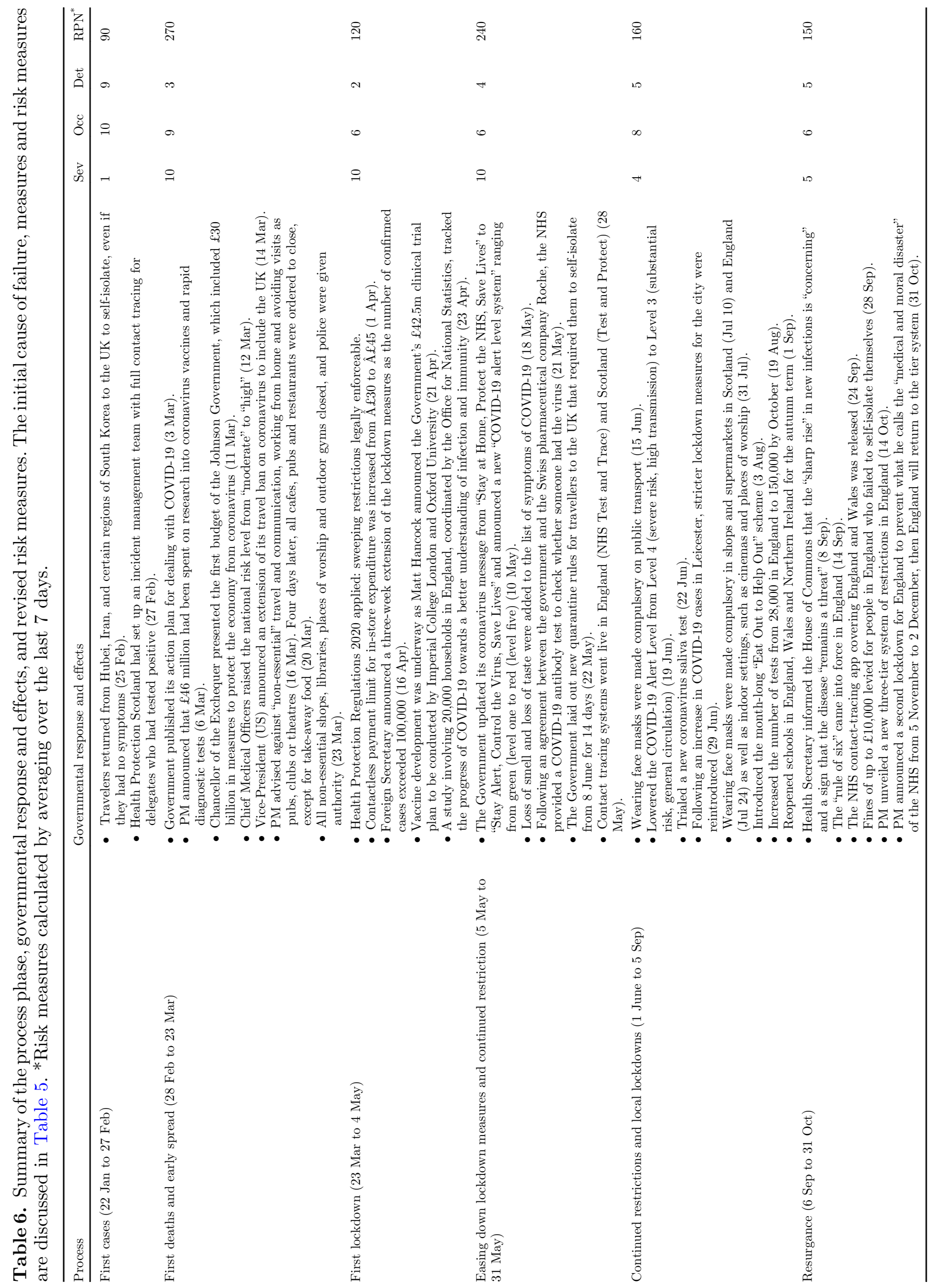


that the posterior estimates of the effective reproduction number for the COVID-19 pandemic in the United Kingdom, under the new model, strongly agrees with the deduced empirical values (as seen in Fig. 6). We then used our FMEA of the community spread of COVID-19 in the United Kingdom and calculated the initial and revised RPN values, where each risk measure was ranked from 1 (insignificant / remote / failure prevented) to 10 (critical/ extremely high/no detection opportunity). For the analysis of the community spread of COVID-19 in the United Kingdom (from March to October 2020), we matched Criteria S (CFR rate) to Figure 8, Criteria O (posterior median of $\mathrm{R}_{t}$ ) to Figure 6 , and Criteria $\mathrm{D}$ (control methods) to the government policies defined in Table 3 for the given time intervals. We found that the initial and revised RPN values seemed to accurately represent the government's contribution to reducing infection rates. For example, in the fourth phase, we found that the government's scientific guidance indicated that the reproduction number had risen from $0.5-0.9$ at the end of the lockdown to $0.7-1.0$ on 15 May, closer to the point at which infections will begin to exponentially escalate. Fittingly, these figures corresponded to our posterior median estimates of $0.66-0.96$ and $0.88-0.94$ for the SEIQR model, respectively.

Although the methodology presented here is intended to contribute to scientific research on Bayesian inference and risk assessment of the current COVID-19 pandemic using FMEA with the modified SEIR model, it can be also used in future pandemics and in wider quality engineering applications.

Acknowledgement. This research was supported by Brunel University London.

\section{References}

1. WHO, Naming the coronavirus disease (COVID-19) and the virus that causes it (2020) https://www.who.int/emergen cies/diseases/novel-coronavirus-2019/technical-guidance/ namingthe-coronavirus-disease-(covid-2019)-and-the-virusthat-causes-it

2. D.S. Hui et al., The continuing 2019-nCoV epidemic threat of novel coronaviruses to global health - the latest2019 novel coronavirus outbreak in Wuhan, China, Int. J. Infectious Dis. 91, 264-266 (2020)

3. R. Breban, J. Riou, A. Fontanet, Interhuman transmissibility of Middle East respiratory syndrome coronavirus: estimation of pandemic risk, The Lancet 382, 694-699 (2013)

4. A. Cheng, Faculty of 1000 evaluation for Pandemic potential of a strain of influenza A (H1N1): early findings, in F1000 Post-publication peer review of the biomedical literature 324.5934 (2009) 1557-1561

5. H.-J. Chang, Evaluation of the basic reproduction number of MERS-CoV during the2015 outbreak in South Korea, in 2016 16th International Conference on Control, Automation and Systems (ICCAS) (2016). DOI: 10.1109/iccas.2016.7832428

6 . R. Ke et al., Estimating the reproductive number R0 of SARS-CoV-2 in the United States and eight European countries and implications for vaccination (2020) DOI: $10.1101 / 2020.07 .31 .20166298$
7. C. Rinzivillo et al., Estimation of the R0 factor of the SIR Model in Italy and some its regions as consequence of the pandemic Sars-Covid-2 (2020) DOI: 10.21203/rs.3.rs-34627/v1

8. M. D'arienzo, A. Coniglio, Assessment of the SARS-CoV-2 basic reproduction number, R0, based on the early phase of COVID-19 outbreak in Italy, Biosaf. Health 2, 57-59 (2020)

9. T. Obadia, R. Haneef, P.-Y. Boëlle, The R0 package: a toolbox to estimate reproduction numbers for epidemic outbreaks, in BMC Medical Informatics and Decision Making 12 (2012) DOI: 10.1186/1472-6947-12-147

10. Imperial College London, Impact of non-pharmaceutical interventions (NPIs) to reduce COVID19 mortality and healthcare demand (2020) https://www.imperial.ac.uk/me dia/imperial-college/medicine/sph/ide/gida-fellowships/Im perial-College-COVID19-NPImodelling-16-03-2020.pdf

11. R. Cuffe, C. Jeavans, How the UK's coronavirus epidemic compares to other countries (2020) https://www.bbc.co.uk/ news/health-52056111

12. S. Sanche et al., High contagiousness and rapid spread of severe acute respiratory syndrome coronavirus 2, Emerg. Infect. Dis. 26 (2020)

13. N. Williams, Social distancing in the covid-19 pandemic, Occupat. Med. (2020) 10.1093/occmed/kqaa072

14. World Health Organization, China Joint Mission on Coronavirus Disease 2019 (COVID-19) (2020) https:// www.who.int/docs/default-source/coronaviruse/who-chinajoint-mission-oncovid-19-final-report.pdf

15. Q. Gao et al., The epidemiological characteristics of 2019 novel coronavirus diseases (COVID-19) in Jingmen, Hubei, China (2020) 113-122. DOI: 10.1101/2020.03.07.20031393.

16. Data of Confirmed Cases on COVID-19. 2020. http://www. nhc.gov.cn/xcs/xxgzbd/gzbd index.shtml

17. GOV.UK. Coronavirus (COVID-19) in the UK. June 2020. https://coronavirus.data.gov.uk/

18. C. Bezier, G. Anthoine, A. Charki, Reliability of real-time RT-PCR tests to detect SARS-Cov-2: a literature review, Int. J. Metrol. Qual. Eng. 11, 16 (2020)

19. C. Bezier, G. Anthoine, A. Charki, Reliability of RT-PCR tests to detect SARSCoV-2: risk analysis, Int. J. Metrol. Qual. Eng. 11, 15 (2020)

20. R.M. Anderson, R.M. May, Infectious diseases of humans: dynamics and control (Oxford University Press, 2010)

21. I. Nesteruk, Statistics based predictions of coronavirus 2019nCoV spreading in mainland China (2020). DOI: 10.1101/ 2020.02.12.20021931

22. O. Diekmann, J.a.p. Heesterbeek, J.a.j. Metz, On the definition and the computation of the basic reproduction ratio $\mathrm{R} 0$ in models for infectious diseases in heterogeneous populations, J. Math. Biol. 28 (1990)

23. C. Castillo-Chavez, Z. Feng, W. Huang, On the Computation of R 0 and its role on global stability, Math. Approach. Emerg. Reemerging Infectious Diseases 229-250 (2002)

24. M. Jit, P. White, Economic analysis of interventions against infectious diseases, Infect. Disease Epidemiol. 243-256 (2016)

25. P.J. White, Mathematical models in infectious disease epidemiology, Infect. Dis. (2017) 10.1016/b978-0-70206285-8.00005-8

26. O. Diekmann, J.A.P. Heesterbeek, Mathematical epidemiology of infectious diseases: model building, analysis and interpretation (Wiley, 2000)

27. P. Van Den Driessche, J. Watmough, Reproduction numbers and sub-threshold endemic equilibria for compartmental models of disease transmission, Math. Biosci. 180, 29-48 (2002) 
28. L.M.A. Bettencourt, R.M. Ribeiro, Real time Bayesian estimation of the epidemic potential of emerging infectious diseases, PLoS ONE 3 (2008)

29. K. Systrom, The MetricWe Need to Manage COVID-19 (2020) http://systrom.com/blog/themetric-we-need-to-man age-covid-19/

30. Worldometer. Coronavirus Incubation Period: 2020. https:// www.worldometers.info/coronavirus/coronavirus-incuba tion-period/

31. COVID Sympton Study. How long does COVID-19 last? June 2020. https:/ covid.joinzoe.com/post/covid-long-term.

32. M. Roser et al., Coronavirus Pandemic (COVID-19) Statistics and Research (2020) https://ourworldindata.org/ coronavirus
33. Imperial College London and I. Mori, Real-time Assessment of Community Transmission findings (2020) https://www.imperi al.ac.uk/medicine/research-and-impact/groups/react-study/ realtime-assessment-of-community-transmission-findings/

34. H. Sjödin et al., COVID-19 healthcare demand and mortality in Sweden in response to non-pharmaceutical mitigation and suppression scenarios, Int. J. Epidemiol. (2020). DOI: $10.1093 / \mathrm{ije} /$ dyaa121

35. V. Chalmers, NHS used HALF as many ICU beds during Covid-19 crisis in the spring as France (2020) https://www. dailymail.co.uk/news/article-8879633/NHS-used-HALFICU-beds-Covid-19-crisis-spring-France.html

36. NHS, The NHS and healthcare in Europe, Eur. Heart J. 39, 3923-3924 (2018)

Cite this article as: Yacine Koucha, QingPing Yang, A Bayesian risk assessment of the COVID-19 pandemic using FMEA and a modified SEIR epidemic model, Int. J. Metrol. Qual. Eng. 12, 14 (2021) 